\title{
Comparison of Diagnostic Accuracy of Active B12 (Holotranscobalamin) and Total Vitamin B12 (Cobalamin) and Verification of Active B12 Test
}

\author{
Barnali Das ${ }^{1}$, Seema Y Bhatia ${ }^{2}$, Poonam M Pal ${ }^{3}$
}

\begin{abstract}
Objective: This study is based on the comparison of the diagnostic accuracy of active B12 (holotranscobalamin) to total vitamin B12 (cobalamin) and verification of the active B12 test for clinical use.

Materials and methods: We have considered 310 individuals (total, mean age $48 \pm 15 y e a r s)$ that includes 233 individuals with serum active B12 levels within the biological reference interval of $20.6-196.7 \mathrm{pmol} / \mathrm{L}$ (controls, mean age $47 \pm 15$ years) and 54 individuals with serum active B12 levels below $20.6 \mathrm{pmol} / \mathrm{L}$ (cases, mean age $46 \pm 13$ years).

Results: Based on the study, we have found that the diagnostic accuracy of active B12 (holotranscobalamin) was found to be better than that of total vitamin B12 (cobalamin). The precision of active B12 testing was found to be comparable to the manufacturer's claim. The reference interval for active B12 was also found to be comparable to the manufacturer's claim.

Conclusion: From the above study, active B12 assay was found to be a better means for detecting vitamin B12 deficiency in individuals, and also, this assay was found to be a better one to follow-up the vitamin B12 levels in the patients who are already vitamin B12-deficient.

Clinical significance: Active B12 (holotranscobalamin) testing can be used as a secondary choice of test for the patients with total B12 levels within the gray-zone $(100-400 \mathrm{pg} / \mathrm{mL})$ or can be used as a primary choice of test for the diagnosis of vitamin B12 deficiency and the follow-up during its treatment.

Keywords: Active B12, Cobalamin, Diagnostic accuracy, Holotranscobalamin, Total B12, Verification, Vitamin B12.

Indian Journal of Medical Biochemistry (2020): 10.5005/jp-journals-10054-0133
\end{abstract}

\section{INTRODUCTION}

Vitamin B12 deficiency is a serious health issue among the elderly population. ${ }^{1}$ It is no longer a rare kind of disease; it prevails as a common condition in approximately $15 \%$ of the elderly population. ${ }^{2}$ Vegetarians mainly suffer from vitamin B12 deficiency for the reason that natural source of vitamin B12 mainly comprises of food from the animal origin. ${ }^{3,4}$ This vitamin B12 deficiency may occur due to malabsorption, prolonged insufficient intake, increased requirements (pregnancy, hyperthyroidism), or excessive loss of vitamin. ${ }^{4,5}$ Malabsorption of vitamin B12 can be drug-induced, protein-bound cobalamin malabsorption, dysfunction of intrinsic factors, pancreatic insufficiency, ileal disease, or pernicious anemia. Also, the patients undergoing gastrectomy have shown a high risk of vitamin B12 deficiency. ${ }^{6,7}$ Small intestinal disorders like celiac disease, ileitis, and Crohn's disease lead to malabsorption of vitamin B12. ${ }^{2}$ Congenital transcobalamin II deficiency also leads to impaired utilization of vitamin B12, which in turn leads to its deficiency. ${ }^{7}$ Vitamin B12 deficiency is found to be associated with neurological deficits like macrocytic anemia and pernicious anemia. ${ }^{8-11}$ Vitamin B12 levels also play a role in cancer patients as in cellular processes, vitamin B12 acts as a coenzyme. These processes include regenerating methionine for protein synthesis, DNA synthesis, and DNA methylation. ${ }^{12}$

Vitamin B12 (cobalamin) when in circulation binds to two proteins, namely haptocorrin (HC) and transcobalamin (TC). The complex formed by cobalamin and transcobalamin protein is known as HoloTC, which is around $10-30 \%$ of total cobalamin. This HoloTC fraction is the active fraction of cobalamin which is taken
${ }^{1-3}$ Department of Laboratory Medicine, Kokilaben Dhirubhai Ambani
Hospital and Medical Research Institute, Mumbai, Maharashtra, India
Corresponding Author: Barnali Das, Department of Laboratory
Medicine, Kokilaben Dhirubhai Ambani Hospital and Medical Research
Institute, Mumbai, Maharashtra, India, Phone: +91-22-30970734,
e-mail: drbarnalid@gmail.com

How to cite this article: Das B, Bhatia SY, Pal PM. Comparison of Diagnostic Accuracy of Active B12 (Holotranscobalamin) and Total Vitamin B12 (Cobalamin) and Verification of Active B12 Test. Indian J Med Biochem 2020;24(1):25-31.

Source of support: Nil

Conflict of interest: None

up by the cells and made available for various biological functions. The rest $80-90 \%$ of total cobalamin bound to haptocorrin forming a complex known as HoloHC has no known biological function and is said to be inert. ${ }^{5,7,13}$ This inert form of vitamin B12 is stored in the liver. ${ }^{14}$ Based on the fact that the active form of cobalamin utilized by the cell is HoloTC, it forms a sensitive marker for detecting vitamin B12 (cobalamin) deficiency. ${ }^{13}$

It is seen that the specificity and sensitivity of vitamin B12 are insufficient for the diagnosis of vitamin B12 deficiency. ${ }^{15}$ Detection of total serum vitamin B12 (cobalamin) can also lead to false results, the main reason being that total serum cobalamin includes that biologically active fraction (HoloTC) as well as the fraction which is not utilized by the cells, that is, inert (HoloHC). Hence, HoloTC (active B12) detection is considered as a reliable approach for detecting 
vitamin B12 deficiency and is more clinically meaningful as it detects only the fraction that is biologically active in the system., ${ }^{1,16}$

In this study, we have compared the HoloTC (active B12) levels in the serum to the total serum cobalamin (vitamin B12) and have suggested the use of HoloTC (active B12) assay as a clinical approach for detecting vitamin B12 deficiency in the patients.

\section{Materials and Methods}

\section{Study Design}

This retrospective study was conducted in the Department Biochemistry and Immunology of Kokilaben Dhirubhai Ambani Hospital, Mumbai, India. A total of 310 individuals between 2 years and 85 years of age (mean age $48 \pm 15$ years) were considered that comprised of 233 individuals (controls) with serum active B12 levels within the biological reference interval (according to the Abbott Diagnostics, Abbott Park, IL, USA, kit used) of 20.6-196.7 $\mathrm{pmol} / \mathrm{L}$ (mean age $47 \pm 15$ years) and 54 individuals (cases) with serum active B12 levels below $20.6 \mathrm{pmol} / \mathrm{L}$ (mean age $47 \pm 13$ ). This complete group of 310 subjects considered for this study included 149 males and 161 females. The parameters taken into consideration include total B12 (pg/mL) and active B12 (pmol/L). Analytical accuracy of active B12 assay was checked by running 20 samples in ARCHITECT i2000 SR as well as ARCHITECT i1000 SR followed by the comparison of the data obtained to check linearity. The verification of the precision of active B12 was carried out according to the CLSI protocol.

\section{Ethical Consideration}

The data used in the study are retrospective data.

\section{Laboratory Methods}

The total B12 concentration in the serum was measured using commercially available ARCHITECT system B12 kits (Abbott Diagnostics, Abbott Park, IL, USA). Similarly, active B12 concentration in the serum was estimated using the commercially available ARCHITECT system active B12 (holotranscobalamin) kits (Abbott Diagnostics, Abbott Park, IL, USA).

\section{Statistical Analysis}

The analysis of the data was done using Minitab (Minitab Inc, PA) and Prism (Graph pad Software, San Diego, CA) statistical software packages. To determine the significance of the data, $t$-test was carried out. This $t$-test can be applied to the data that have normal distribution or to the data that have lognormal distribution; hence, normal distribution of data was checked by the histogram with fit curve and was confirmed by probability plots. F-test was applied to each of the pairs of case and control groups for total B12 and active $\mathrm{B} 12$ to determine whether the variance between case and control groups for each was significant. Also, modified $t$-test with Welch's correlation was applied to the case and control groups for total B12 as well as active B12.

Cumulative distribution function was applied to the case and control groups of both, total B12 and active B12 to estimate the percentage of the population mistakenly classified as controls. The cumulative distribution function (CDF) calculates the cumulative probability for a given X-value. The CDF is used to determine the probability that a random observation that is taken from the population will be less than or equal to a certain value or the observation will be greater than a certain value, or between two values. It helps to find out the difference in the value of the case and the control groups. This difference is independent of the external factors like lifestyle, environmental factors, etc.

Receiver operating characteristic (ROC) curve analysis was also carried out for case and control for total B12 and active B12 to determine the accuracy of the test. The accuracy of the test is determined on the basis of the area under the curve (AUC).

Linear regression analysis was applied to the data for active B12 assay to check analytical accuracy and coefficient of determinant $\left(r^{2}\right)$ along with correlation coefficient $(r)$ was calculated.

\section{Results}

The total subjects (310 individuals) considered for the study were bifurcated into two groups, namely case and control, based on the serum active B12 concentration. The case group (54 individuals) consisted of individuals with active B12 concentration below 20.6 $\mathrm{pmol} / \mathrm{L}$, whereas the control group (233 individuals) consisted of individuals with active $\mathrm{B} 12$ concentration in the biological reference interval of 20.6-196.7 pmol/L. From the total subjects, 23 individuals had the active B12 levels more than $256 \mathrm{pmol} / \mathrm{L}$; this population was not considered for the case group as well as the control group.

The data obtained on estimating the serum active B12 and total B12 concentration in 310 individuals are shown below (Table 1).

The total B12 and active B12 levels were compared using a scatter diagram (Fig. 1) for 310 subjects considered. The gray zone was marked for total B12 serum concentration. This gray zone indicates that the total B12 serum concentrations of the symptomatic patients lying in this interval are suggested further testing. The gray zone for total B12 lies between 100 and $400 \mathrm{pg} / \mathrm{mL}^{7}$

As mentioned that prior to the application of $t$-test for determining the significance of the data, it is necessary to check the normal distribution of the data, histogram with a fit curve was plotted for control and case groups (Fig. 2). This normal distribution was further confirmed with probability plots for control and case groups (Fig. 3). The histogram plots (Fig. 2) indicate a normal distribution of data for control and case groups, shown by the continuous curve, whereas the probability plots show that the data lie within $95 \%$ confidence interval $(\mathrm{Cl})$ of lognormal fit.

The CDF analysis was carried out for active B12 (control and case groups) as well as for total B12 (control and case groups) to determine the percentage of the population in case group that mistakenly was classified as controls. The $x$-axis shows the active B12 and total B12 data, as observed in case group, whereas the $y$-axis gives the percentage of samples having active B12 and total $B 12$ values below that. This graph gives a value of maximum vertical deviation between the plotted curves. Greater the value of deviation, greater is the separation between the groups. The point of overlap of the curves determines the percentage of the population mistakenly classified as control. Figure 4 shows the CDF analysis for active B12 and total B12. The CDF analysis of active B12 indicates that $20.6 \%$ of the case group population could have mistakenly been classified as controls, whereas in case of total B12 CDF analysis, $36.02 \%$ of the case group were mistakenly classified as controls.

The total data for active B12 and total B12 were subjected to ROC curve analysis to check the ability of active B12 and total B12 assays to predict the population likely of having metabolic vitamin B12 deficiency (Fig. 5). The ROC curve plots true-positive 
Table 1: Data representation: levels of active B12 and total B12

\begin{tabular}{llllll}
\hline & \multicolumn{2}{c}{ Active B12 $(\mathrm{pmol} / \mathrm{L})$} & & \multicolumn{2}{c}{ Total B12 $(\mathrm{pg} / \mathrm{mL})$} \\
\cline { 2 - 3 } \cline { 6 - 7 } & Control $(n=233)$ & Case $(n=54)$ & & Control $(n=233)$ & Case $(n=54)$ \\
\hline Mean \pm SD & $55.13 \pm 33.75$ & $15.32 \pm 3.86$ & & $273.84 \pm 141.30$ & $182.70 \pm 50.74$ \\
Quartile (Q1-Q3) & $31.35-70.1$ & $12.42-18.3$ & & $190.75-316.45$ & $149.52-204.75$ \\
$p$ value & $<0.0001(\mathrm{~S})$ & $<0.0001(\mathrm{~S})$ & & $<0.0001(\mathrm{~S})$ & $<0.0001(\mathrm{~S})$ \\
\hline
\end{tabular}

$S$, significant

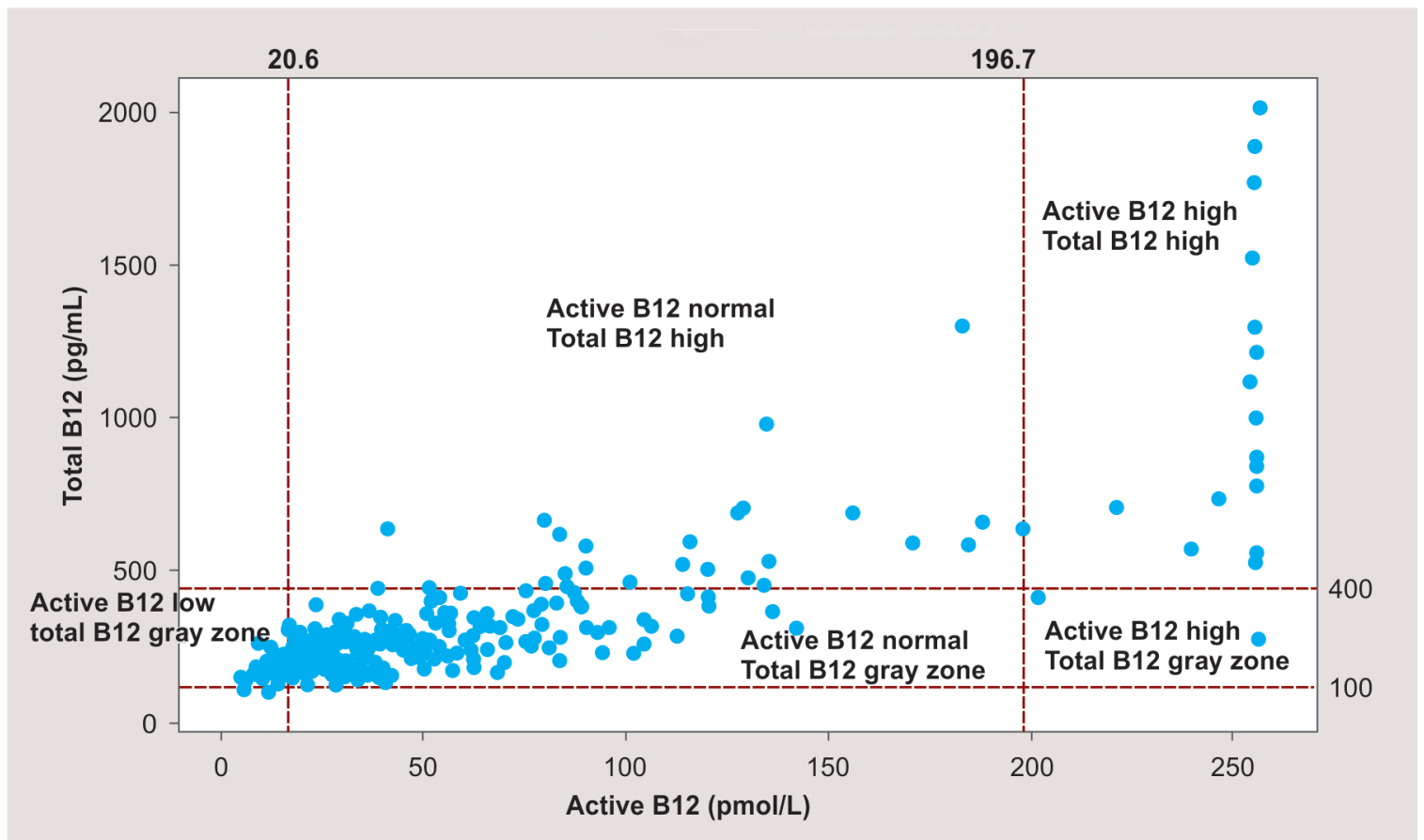

Fig. 1: Scatterplot to compare total B12 and active B12 levels in the study subjects
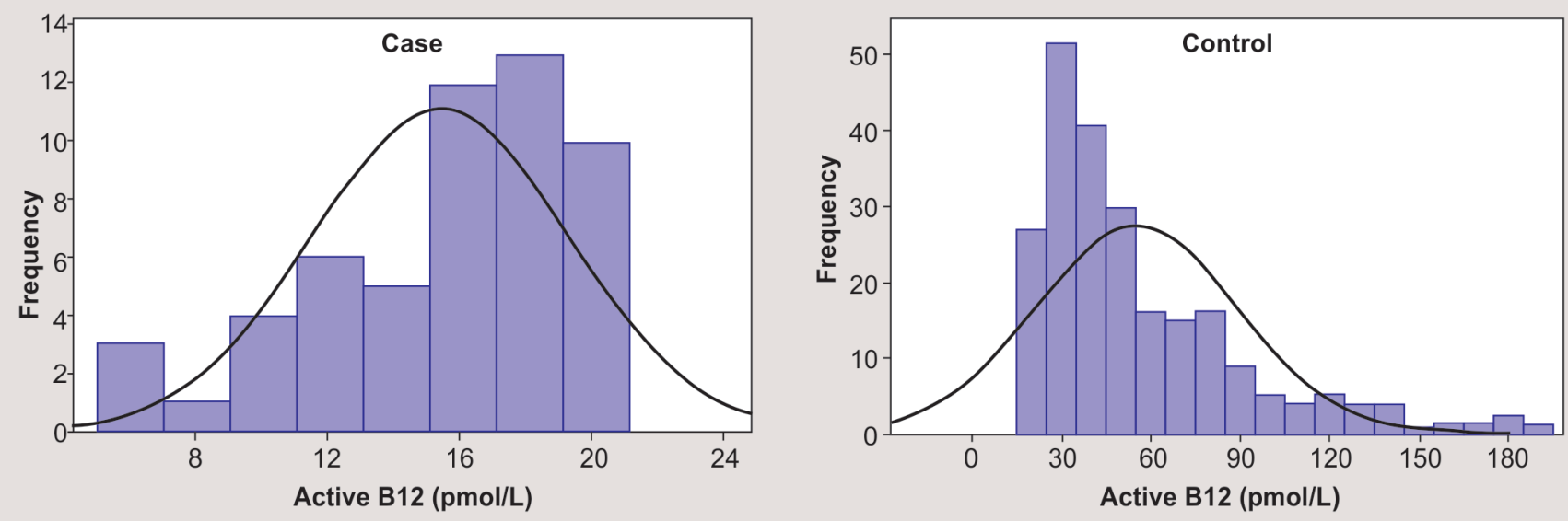

Fig. 2: Histogram of active B12 data and corresponding log-normal fit for case and control groups

vs false-positive for different cut points in the diagnostic test. The test accuracy depends on the AUC.

The AUC of $0.90-1$ indicates that the diagnostic test has an excellent accuracy, $0.80-0.90$ indicates a good accuracy, $0.70-0.80$ indicates that the diagnostic test is fairly accurate, $0.60-0.70$ indicates a poor accuracy, and $0.50-0.60$ indicates a failure. From the ROC curve analysis, the AUC for active B12 was found to be 1.0 with $95 \% \mathrm{Cl} 1.0$ to 1.0 , standard error 0.0 , and $p$ value of $<0.0001$, whereas the AUC for total B12 was found to be 0.7346 with $95 \%$ $\mathrm{Cl} 0.6928$ to 0.8234 , standard error 0.0333 , and $p$ value of $<0.0001$. 

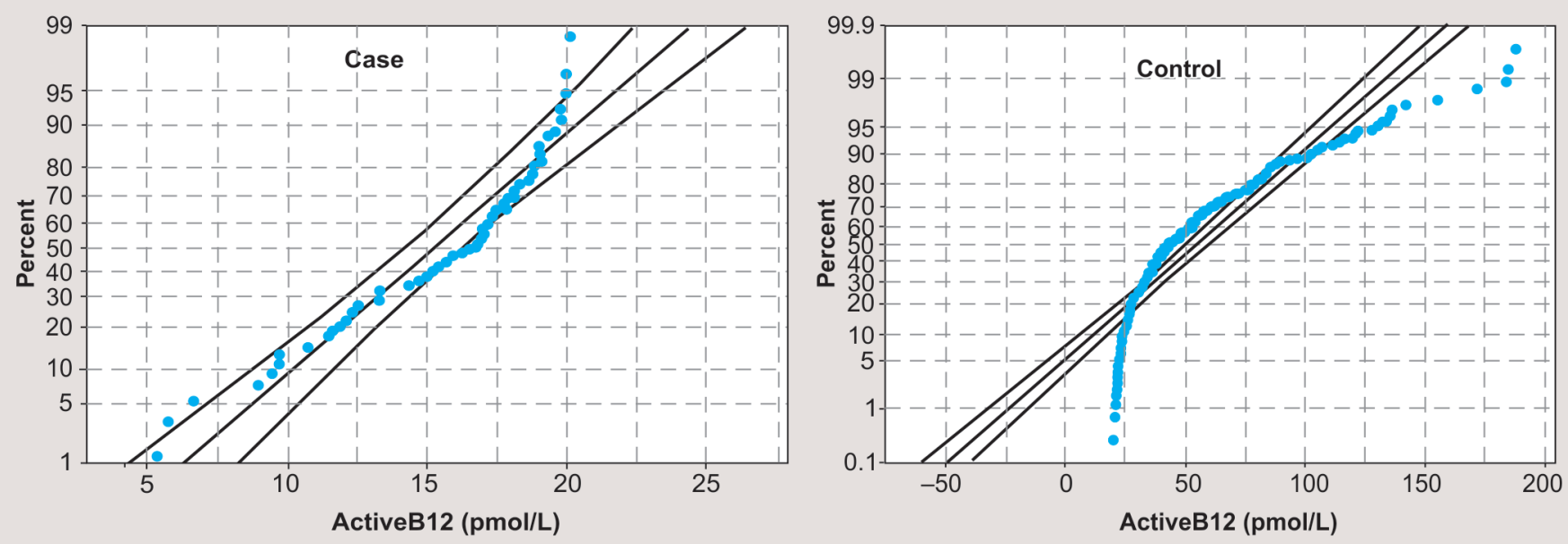

Fig. 3: Probability plot of active B12 data and corresponding normal fit for case and control groups
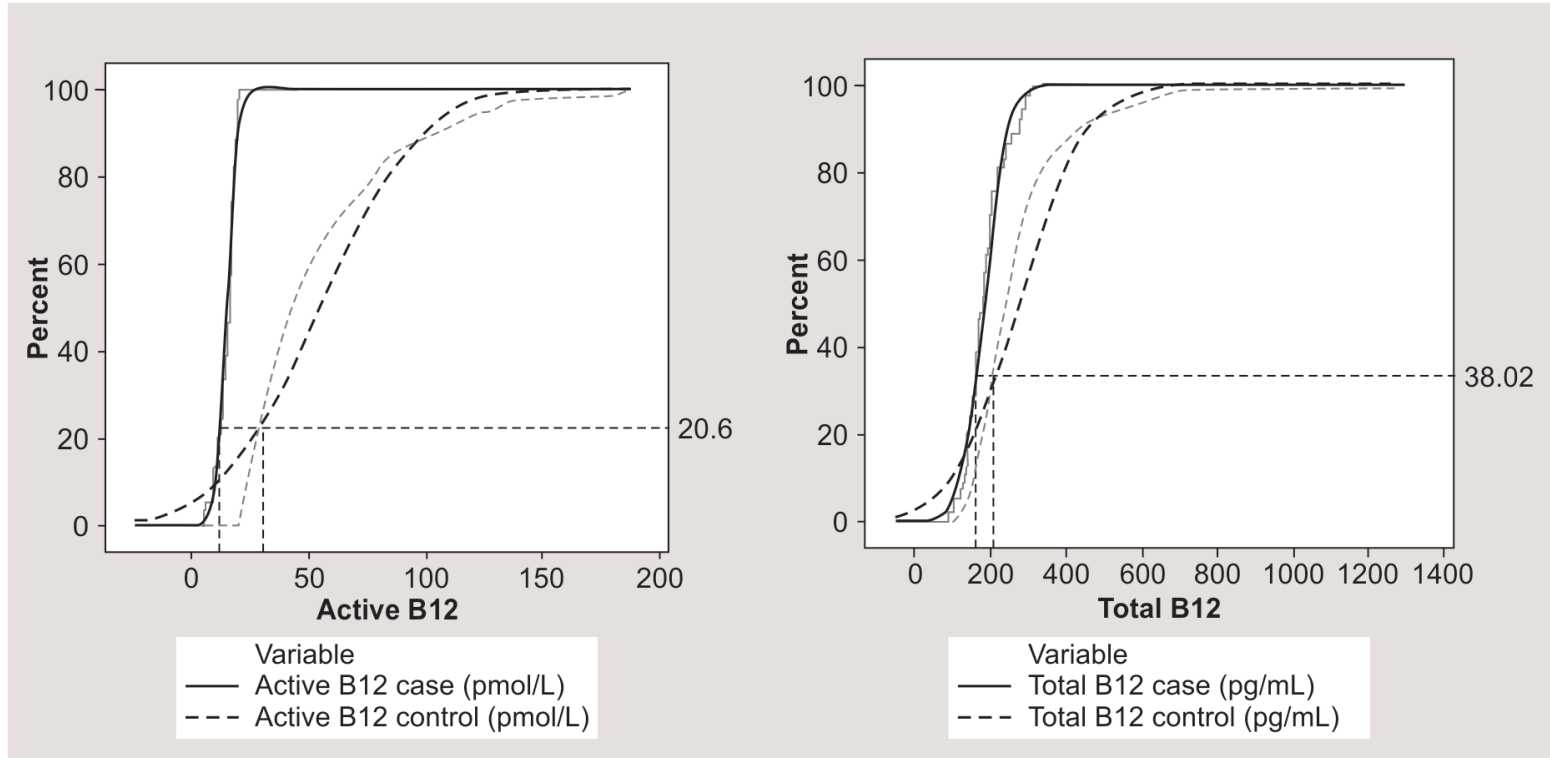

Fig. 4: Cumulative distribution function (CDF) plots of active B12 and total B12
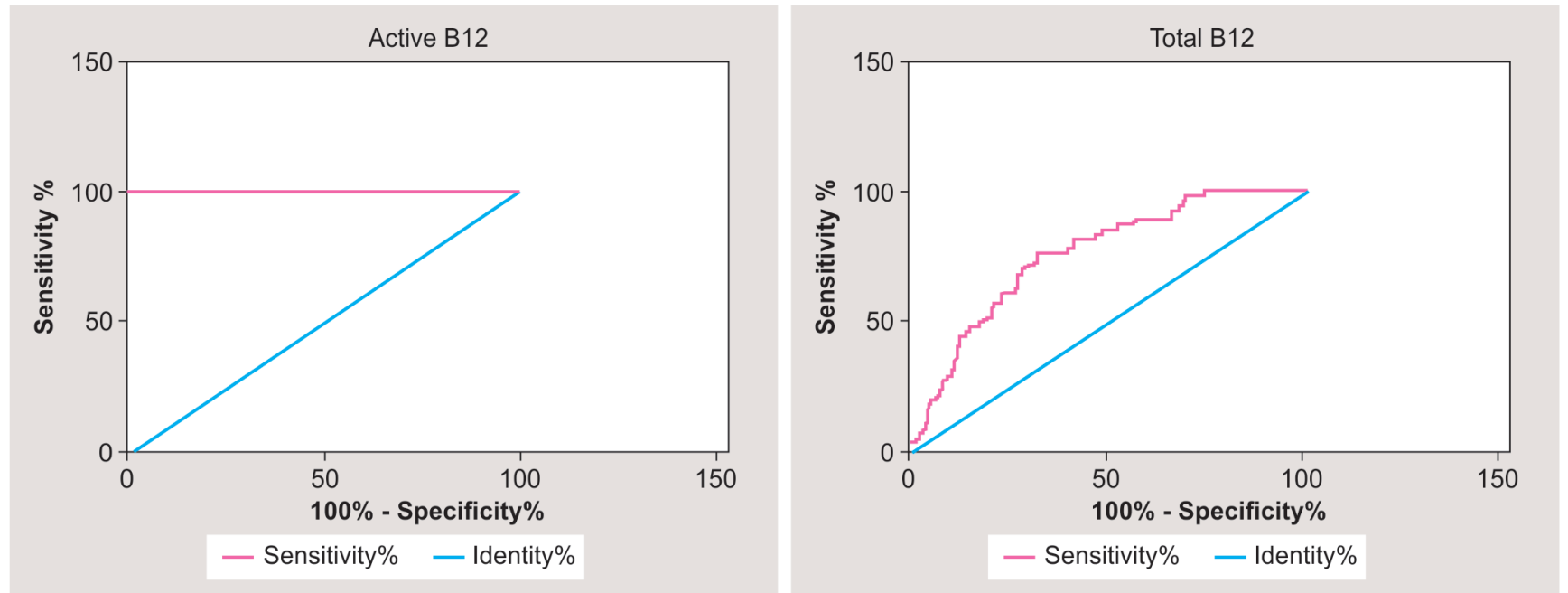

Fig. 5: Receiver operating characteristic curve (ROC) of active B12 and total B12 


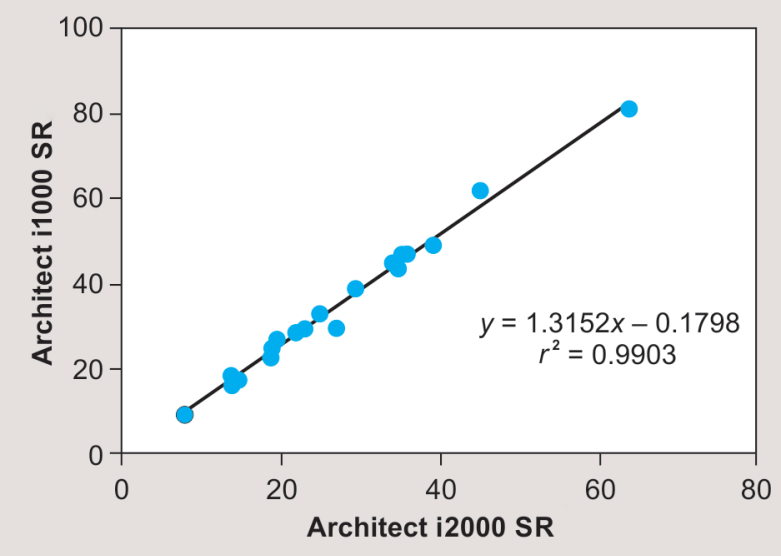

Fig. 6: Linear regression analysis plot of active B12 data for accuracy verification

Table 2: Analytical accuracy of active B12 assay

\begin{tabular}{lll}
\hline & Levels of active B12 (pmol/L) \\
\hline Sample no. & Architect i2000 SR & Architect i1000 SR \\
\hline 1 & 19.4 & 27.3 \\
2 & 21.9 & 28.7 \\
3 & 29.1 & 39.1 \\
4 & 19 & 25.4 \\
5 & 13.7 & 18.7 \\
6 & 35.8 & 47.6 \\
7 & 34.9 & 47.7 \\
8 & 34.3 & 44 \\
9 & 22.8 & 29.7 \\
10 & 24.8 & 33.1 \\
11 & 13.5 & 16.7 \\
12 & 44.7 & 62.1 \\
13 & 7.7 & 9.6 \\
14 & 33.7 & 45.2 \\
15 & 63.7 & 81.4 \\
16 & 38.9 & 49.7 \\
17 & 18.6 & 23.3 \\
18 & 14.4 & 17.7 \\
19 & 18.6 & 24.2 \\
20 & 26.6 & 30.3 \\
\hline
\end{tabular}

\section{Verification Analysis}

According to CAP and NABL, ISO 15189, CLIA requirements the data produced in the laboratory should be verified on its accuracy, precision, and reference interval. The data obtained for analytical accuracy check for active B12 were subjected to linear regression analysis to check its reliability (Fig. 6). On carrying out the analysis, the data were found to be reliable with the regression coefficient $\left(r^{2}\right)$ of 0.9903 and the correlation coefficient $(r)$ as 0.9951 .

To check the analytical accuracy of the test, 20 samples within the analytical measurable range (AMR) were analyzed by both new and comparative methods. The AMR is the range that can be reported without the dilution of the samples. The data for the analytical accuracy of active B12 are shown in Table 2.

For precision study, five replicates per control were analyzed for five days. Table 3 represents the data for precision study for active B12.
Reference interval was determined by considering the active B12 data obtained from 161 healthy individuals. On the data analysis, the reference interval for active B12 was found to be 20.6-104.4 pmol/L which was satisfying when compared to the reference interval claimed by the manufacturer which was 25.1-165 pmol/L.

\section{Discussion}

In this study, we have given importance to deficiency of vitamin B12, which is no longer considered as a rare disorder and has become a common scenario. ${ }^{2}$ The association of vitamin B12 deficiency to neurological disorders, trigeminal neuralgia, end-stage renal disorders and patient having undergone gastrectomy makes it a matter of great importance. .,9 $^{\circ}$

We have targeted the ongoing total B12 assay, which is used widely for the detection of vitamin B12 concentration in an individual and have compared it to active B12 assay used for the detection of the same. We aim at choosing a better diagnostic method for detection of vitamin B12 levels in the serum to avoid false reporting and for better diagnosis of vitamin B12 deficiency.

Based on our results, we found that the diagnostic accuracy of activeB12 assay was better than that of total B12 assay, which is used at present for the detection of vitamin B12 and in the follow-up of the vitamin B12 levels in case of vitamin B12-deficient patients. Also, the precision of active B12 testing was also found to be comparable to the manufacturer's claimed. Both withinrun precision and within-laboratory precision of active B12 assay were found to be comparable to the manufacturer's claim. The reference interval established by considering the active B12 data of 161 healthy individuals was found to be comparable to the manufacturer's claim.

We believe that the study has clinical relevance as it avoids false reporting. We also believe that there has been no Indian study which has given a comparison of active B12 and total B12 assays on the bases of diagnostic accuracy and precision. ${ }^{17}$ It is known that in spite of having indicative markers like methylmalonic acid (MMA) and homocysteine (Hcy) being related to vitamin B12 deficiency, there was a need for developing an accurate marker for detecting vitamin B12 deficiency due to the limitations associated with these markers. As known from the previous studies, the MMA levels are affected during a number of clinical conditions like renal dysfunction, genetic disorder, antibiotic therapy, etc. Similarly, the Hcy levels are also affected by renal dysfunction, folate deficiency, genetic disorder, etc. ${ }^{7}$ Hence, the specificity of MMA and Hcy in the context of vitamin B12 deficiency is debatable and this has been majorly seen in the case of elderly population with renal dysfunction. ${ }^{1}$ It has been seen that total B12 assay suffers from false results due to the reasons like folate deficiency, liver disorder, etc. $^{7}$ As the only fraction of vitamin B12 utilized by the cells for its biological function is the one linked to holotranscobalamin (HoloTC) or active B12, it is clinically meaningful to detect the active fraction of vitamin B12. Therefore, active B12 or HoloTC have higher sensitivity and specificity for diagnosis and treatment of vitamin B12 deficiency. $7,18,19$

\section{Conclusion}

Since the accuracy, reliability, and efficiency of active B12 assay were found to be higher as compared to those of total B12 assay, using active B12 approach is a good choice for diagnosis as well as prognosis. 
Table 3: Precision (repeatability) for active B12

\begin{tabular}{|c|c|c|c|c|c|c|c|c|}
\hline & \multicolumn{5}{|c|}{ Low control (L) } & \multicolumn{3}{|c|}{ High control $(H)$} \\
\hline \multicolumn{2}{|l|}{ Concentration target } & \multicolumn{3}{|c|}{15} & & \multicolumn{3}{|c|}{48} \\
\hline \multicolumn{2}{|l|}{ Concentration range } & \multicolumn{3}{|c|}{$9.6-21.8$} & & \multicolumn{3}{|c|}{$29.6-66.7$} \\
\hline \multicolumn{9}{|l|}{ Day 1} \\
\hline Sample ID & Rep 1 & Rep2 & Rep3 & Rep 4 & Rep 5 & Mean & SD & CV\% \\
\hline Observed value $(L)$ & 14.9 & 14.3 & 14.4 & 14.3 & 14.2 & 14.42 & 0.27749 & 1.92433 \\
\hline Observed value $(H)$ & 48.2 & 47.4 & 47.4 & 48 & 47 & 47.6 & 0.4899 & 1.0292 \\
\hline \multicolumn{9}{|l|}{ Day 2} \\
\hline Sample ID & Rep 1 & Rep2 & Rep3 & Rep 4 & Rep 5 & Mean & SD & CV\% \\
\hline Observed value $(L)$ & 12.3 & 13 & 12.3 & 12.6 & 12.3 & 12.5 & 0.308 & 2.46577 \\
\hline Observed value $(H)$ & 42.3 & 41.1 & 41.1 & 40.8 & 40.2 & 41.1 & 0.76485 & 1.86096 \\
\hline \multicolumn{9}{|l|}{ Day 3} \\
\hline Sample ID & Rep 1 & Rep2 & Rep3 & Rep 4 & Rep 5 & Mean & SD & CV\% \\
\hline Observed value $(L)$ & 13.8 & 13.4 & 13.3 & 13.1 & 13 & 13.32 & 0.31145 & 2.3382 \\
\hline Observed value $(H)$ & 43.9 & 41.8 & 42.2 & 42.8 & 41.6 & 42.46 & 0.92628 & 2.18154 \\
\hline \multicolumn{9}{|l|}{ Day 4} \\
\hline Sample ID & Rep 1 & Rep2 & Rep3 & Rep 4 & Rep 5 & Mean & SD & CV\% \\
\hline Observed value $(L)$ & 15.7 & 15.8 & 15.6 & 14.9 & 16 & 15.6 & 0.41833 & 2.6816 \\
\hline Observed value $(H)$ & 48.4 & 47.4 & 46.8 & 46.9 & 46.9 & 47.14 & 0.80498 & 1.70765 \\
\hline \multicolumn{9}{|l|}{ Day 5} \\
\hline Sample ID & Rep 1 & Rep2 & Rep3 & Rep 4 & Rep 5 & Mean & SD & $\mathrm{CV} \%$ \\
\hline Observed value $(L)$ & 11.7 & 11.4 & 11.2 & 11.1 & 11.1 & 11.3 & 0.25496 & 2.2562 \\
\hline Observed value $(H)$ & 37.8 & 37.4 & 37.3 & 35.8 & 36.4 & 36.94 & 0.81731 & 2.21254 \\
\hline
\end{tabular}

where $\mathrm{CV} \%$ = coefficient of variation

\section{Clinical Significance}

As vitamin B12 deficiency is quite common, active B12 assay being more accurate, reliable, and efficient, it can be used as secondary choice of test for the patients with total B12 levels within the gray zone as well as it can be used as a primary choice of test for the diagnosis and treatment of vitamin B12 deficiency.

\section{Acknowledgments}

The author extends a thanks to the Department of Biochemistry and Immunology, Kokilaben Ambani Hospital and Medical Research Institute, Mumbai, India, and Dr Sameer Tulpule and Dr Gaurav Mehta (Kokilaben Ambani Hospital and Medical Research Institute, Mumbai, India), and a special thanks to Mr Karna S Yajnik, MSc Statistics, NMIMS School of Science (2009), Mumbai, India.

\section{Manufacturer}

Abbott Diagnostics, Abbott Park, Illinois, USA; Minitab Inc, PA, USA; GraphPad Software, Inc., San Diego, CA, USA

\section{References}

1. Valente E, Scott JM, Ueland PM, et al. Diagnostic accuracy of holotranscobalamin, methylmalonic acid, serum cobalamin, and other indicators of tissue vitamin B12 status in the elderly. Clin Chem 2011;57(6):856-863. DOI: 10.1373/clinchem.2010.158154.

2. Hvas AM, Nexo E, Diagnosis and treatment of Vitamin B12 deficiencyan, 2006.

3. Herrmann W. Vitamin B-12 status, particularly holotranscobalamin II and methylmalonic acid concentrations, and hyperhomocysteinemia in vegetarians. Am J Clin Nutrit 2003;78(1):131-136. DOI: 10.1093/ ajcn/78.1.131.
4. Herbert V. Staging vitamin status in vegetarians. Am J Clin Nutrit 1994;59:1213. DOI: 10.1093/ajen/59.5.1213S.

5. Herrmann W, Obeid R, Schorr $\mathrm{H}$, et al. The usefulness of holotranscobalamin in predicting vitamin B12 status in different clinical settings. Curr Drug-Metabol 2005;6(1):47-53. DOI: 10.2174/1389200052997384.

6. Lee YK, Kim HS, Kang HJ. Holotranscobalamin as an indicator of vitamin B12 deficiency in gastrectomized patients. Ann Clin Lab Sci 2009;39(4):361-366.

7. Snow CF. Diagnosis of vitamin B 12 and folate deficiency. Arch Intern Med 2006.

8. Chararin I. Megaloblastic anemia, cobalamin and folate. J Clin Pathol 1987;40(9):978-984. DOI: 10.1136/jcp.40.9.978.

9. Aggarwal A, Wood I. Low vitamin B12 syndrome in trigeminal neuralgia. J Pain 2012;1(5):1-4. DOI: 10.4172/2167-0846.1000109.

10. Lai SC, Nakayama Y, Sequeira JM, et al. The transcobalamin receptor knockout mouse: a model for vitamin B12 deficiency in the central nervous system. FASEB J 2013;27(6):2468-2475. DOI: 10.1096/fj.12219055.

11. Abdulmanea AA, Alsaeed AH, Shaik AP, et al. Pernicious anemia in patients with macrocytic anemia in patients with macrocytic anemia and low serum B12. J Med Sci 2014;30(6):1218-1222.

12. Vira HJ, Importance of assessing total vitamin B12 and active B12 levels in cancer patients. 2018.

13. Nexo E, Hvas AM. Holotranscobalamin-first choice assay for diagnosing early vitamin B12 deficiency. J Intern Med Suppl 2005;257(3):289-298. DOI: 10.1111/j.1365-2796.2004.01437.x.

14. Sugihara $T$, Koda $M$, Okamoto $T$, et al. Falsely elevated serum vitamin B12 levels were associated with the severity and prognosis of chronic viral liver disease. Yonago Acta Med 2017;60(1):31-39.

15. Golding PH. Holotranscobalamin (HoloTC, Active-B12) and Herbert's model for the development of vitamin B12 deficiency: a review and alternative hypothesis. Springerplus 2016;5(1):668. DOI: 10.1186/ s40064-016-2252-z. 
16. Nexo E, Hoffmann-Lücke E. Holotranscobalamin, a marker of vitamin B-12 status: analytical aspects and clinical utility. Am J Clin Nutrit 2011;1(5):1-7. DOI: 10.3945/ajcn.111.013458.

17. Al Aisari F, Al-Hashmi H, Mula-Abed W-A. Comparison between serum holotranscobalamin and total vitamin B12 as indicators of vitamin B12 status. Oman Med J 2010;1:9-12.
18. Ulleland M, Eilertsen I, Quadros EV, et al. Direct assay for cobalamin bound to transcobalamin (holo-transcobalamin) in serum. Clin Chem 2002;48(3):526-532. DOI: 10.1093/clinchem/48.3.526.

19. Bagge $E$, Nexo $E$, Lindgren $A$, et al. Holotranscobalamin-a sensitive marker of cobalamin malabsorption. Eur J Clin Invest 1999;29(4): 321-329. DOI: 10.1046/j.1365-2362.1999.00446.x. 